\title{
Transverse momentum distributions inside the nucleon from lattice QCD
}

\author{
Bernhard Musch ${ }^{*}{ }^{a}$ Philipp Hägler, ${ }^{a d}$ John W. Negele ${ }^{c}$ and Andreas Schäfer ${ }^{d}$ \\ ${ }^{a}$ Theory Center, Jefferson Lab, 12000 Jefferson Avenue \\ Newport News, VA 23606, USA \\ ${ }^{b}$ Theoretische Physik T39, TU München, James-Franck-Straße 1, \\ 85747 Garching, Germany \\ ${ }^{c}$ Center for Theoretical Physics, Massachusetts Institute of Technology, \\ 77 Massachusetts Avenue, Bldg. 6-315, Cambridge, MA 02139, USA \\ ${ }^{d}$ Institut für Theoretische Physik, Universität Regensburg, \\ 93040 Regensburg, Germany \\ E-mail: bmuschejlab.org, phaeglereph.tum.de
}

\begin{abstract}
We study transverse momentum dependent parton distribution functions (TMDs) with non-local operators in lattice QCD, using MILC/LHPC lattices. Results obtained with a simplified operator geometry show visible dipole deformations of spin-dependent quark momentum densities. We discuss the basic concepts of the method, including renormalization of the gauge link, and an extension to a more elaborate operator geometry that would allow us to analyze process-dependent TMDs such as the Sivers-function.
\end{abstract}

The XXVIII International Symposium on Lattice Filed Theory

June 14-19,2010

Villasimius, Sardinia Italy

${ }^{*}$ Speaker. 


\section{Introduction}

Generalized parton distribution functions (GPDs) and transverse momentum dependent parton distribution functions (TMDs) provide us with a picture of the internal quark distributions in a nucleon at the instant of an interaction, see illustration Fig. 1 a). GPDs and TMDs have their natural interpretation at large nucleon momentum $\mathbf{P}=\left(0,0, \mathbf{P}_{z}\right)$. The quark momentum $k$ in terms of light cone coordinates $k^{ \pm} \equiv\left(k^{0} \pm k^{3}\right) / \sqrt{2}, \mathbf{k}_{\perp}=\left(\mathbf{k}_{x}, \mathbf{k}_{y}\right)$ scales like $k^{+}: \mathbf{k}_{\perp}: k^{-} \sim P^{+}: 1:\left(P^{+}\right)^{-1}$ with the large momentum component $P^{+}$of the nucleon. TMDs resolve the dependence on $x \equiv k^{+} / P^{+}$ and transverse momentum $\mathbf{k}_{\perp}$, but not on the suppressed component $k^{-}$. In spin-polarized channels at leading twist, TMDs encode dipole- or quadrupole-shaped deformations of the nucleon in the $\mathbf{k}_{\perp^{-}}$ plane. We have studied such deformations in first explorative lattice QCD calculations $[1,2,3]$, see Fig. 1 and our discussion below. These studies have been motivated by a history of successful lattice computations of $x$-moments of GPDs, providing images of the nucleon in the impact parameter, $\mathbf{b}_{\perp^{-}}$, plane, see [4] for a review. A remaining theoretical problem concerns the precise form of the correlator defining TMDs in the continuum, see [5,6] and references therein. In its basic form, it is given by [7]

$$
\begin{aligned}
& \Phi_{q}^{[\Gamma]}\left(x, \mathbf{k}_{\perp} ; P, S ; \mathscr{C}\right) \equiv \\
& \left.\int d k^{-} \int \frac{d^{4} l}{(2 \pi)^{4}} e^{-i k \cdot l} \underbrace{\frac{1}{2}\left\langle P, S\left|\bar{q}(l) \Gamma \mathscr{U}\left[\mathscr{C}_{l}\right] q(0)\right| P, S\right\rangle}_{\widetilde{\Phi}_{q}^{[\Gamma]}(l, P, S ; \mathscr{C})}\right|_{k^{+}=x P^{+}} \\
& =\left.\int \frac{d l^{-}}{2 \pi} e^{-i l^{-} k^{+}} \int \frac{d^{2} \mathbf{l}_{\perp}}{(2 \pi)^{2}} e^{i \mathbf{l}_{\perp} \cdot \mathbf{k}_{\perp}} \widetilde{\Phi}_{q}^{[\Gamma]}(l, P, S ; \mathscr{C})\right|_{l^{+}=0} \\
& =\left.\frac{1}{P^{+}} \underbrace{\int \frac{d(l \cdot P)}{2 \pi} e^{-i(l \cdot P) x}}_{\mathscr{f}} \underbrace{\int \frac{d^{2} \mathbf{l}_{\perp}}{(2 \pi)^{2}} e^{i \mathbf{l}_{\perp} \cdot \mathbf{k}_{\perp}}}_{\mathscr{f}} \widetilde{\Phi}_{q}^{[\Gamma]}(l, P, S ; \mathscr{C})\right|_{l^{+}=0}
\end{aligned}
$$

where $\Gamma$ is a Dirac matrix. The Wilson line $\mathscr{U}\left[\mathscr{C}_{l}\right]$ running along a continuous path $\mathscr{C}_{l}$ from $l$ to 0 ensures gauge invariance of the expression. For the SIDIS and Drell-Yan scattering process, the Wilson line extends to infinity along a direction $v$, see Fig. 4 a) below, such that the cross section factorizes into hard, perturbative parts and soft contributions. Based on its symmetry transformation properties, the above correlator can be parametrized in terms of TMDs $[8,9,10]$, for example

$$
\begin{aligned}
& 2 \rho_{T L}^{(q)} \equiv \Phi_{q}^{\left[\gamma^{+}+\lambda \gamma^{+} \gamma^{5}\right]}=f_{1, q}+\lambda \frac{\mathbf{k}_{\perp} \cdot \mathbf{S}_{\perp}}{m_{N}} g_{1 T, q}+\left[\frac{\mathbf{S}_{j} \varepsilon_{j i} \mathbf{k}_{i}}{m_{N}} f_{1 T, q}^{\perp}\right]_{\text {odd }}, \\
& 2 \rho_{L T}^{(q)} \equiv \Phi_{q}^{\left[\gamma^{+}-s^{j} i \sigma^{+j} \gamma^{5}\right]}=f_{1, q}+\Lambda \frac{\mathbf{k}_{\perp} \cdot \mathbf{s}_{\perp}}{m_{N}} h_{1 L, q}^{\perp}+\left[\frac{\mathbf{s}_{j} \varepsilon_{j i} \mathbf{k}_{i}}{m_{N}} h_{1, q}^{\perp}\right]_{\text {odd }} .
\end{aligned}
$$

Here $\lambda$ and $\mathbf{s}_{\perp}$ are longitudinal and transverse quark polarization, $\Lambda$ and $\mathbf{S}_{\perp}$ longitudinal and transverse nucleon polarization, and the leading-twist TMDs $f_{1, q}, g_{1 T, q}, f_{1 T, q}^{\perp}, h_{1 L, q}^{\perp}$ and $h_{1, q}^{\perp}$ are realvalued functions of $x$ and $\mathbf{k}_{\perp}^{2}$. The "naively time-reversal odd" TMDs $f_{1 T, q}^{\perp}$ and $h_{1, q}^{\perp}$ switch their sign when comparing the SIDIS- with the Drell-Yan process, because the direction $v$ of the Wilson line changes from future- to past-pointing [11]. 
a)

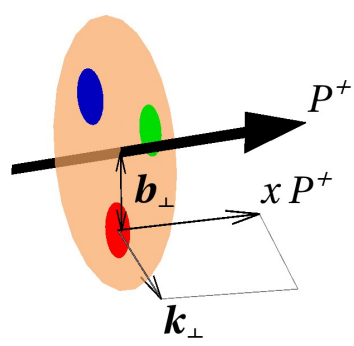

b)

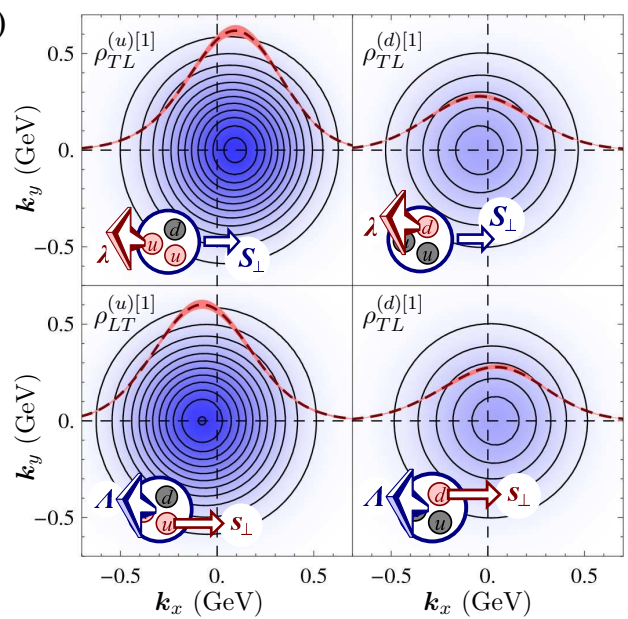

Figure 1: a) Illustration of quark degrees of freedom in the nucleon at large momentum. b) Dipole-deformed $x$-integrated densities obtained with straight gauge links at a pion mass $m_{\pi} \approx 500 \mathrm{MeV}$. The insets display the spin polarization of the quarks (red arrow) and of the nucleon (blue arrow).

a)

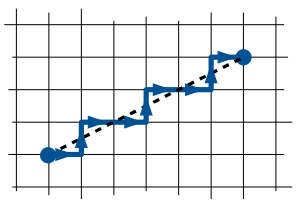

b)

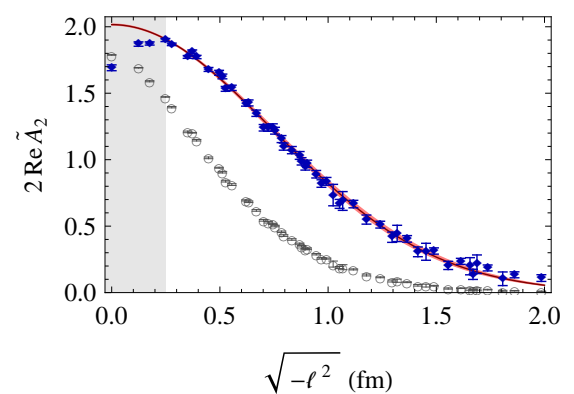

Figure 2: a) Representation of a straight Wilson line (dashed line) as a step-like product of link variables. b) Amplitude $\widetilde{A}_{2}\left(l^{2}, 0\right)$ for up quarks at a pion mass $m_{\pi} \approx 500 \mathrm{MeV}$, using straight gauge links.

\section{Straight link TMDs from the lattice}

In light of the uncertainties about the precise form of the continuum correlator, and to develop our methods, our first lattice studies employ a simple operator geometry that does not relate to a specific scattering process: We connect the quark fields with a direct, straight Wilson line. For the resulting "process-independent" TMDs, the T-odd functions $f_{1 T, q}^{\perp}$ and $h_{1, q}^{\perp}$ vanish exactly.

In our approach, we calculate matrix elements $\langle P, S|O| P, S\rangle$ from ratios of three- and two-point functions using the same techniques as GPD calculations by the LHP collaboration in Ref. [12]. We also use the same sequential propagators and quark propagators, calculated by LHPC with domain-wall valence fermions on top of asqtad-improved staggered MILC gauge configurations $[13,14,15]$ with $2+1$ quark flavors at a lattice spacing $a \approx 0.12 \mathrm{fm}$. The difference with respect to GPD calculations is that we directly insert the non-local operator $O \equiv \bar{q}(l) \Gamma \mathscr{U}\left[\mathscr{C}_{l}\right] q(0)$ in our threepoint function. The Wilson line $\mathscr{U}\left[\mathscr{C}_{l}\right]$ is approximated as a step-like product of HYP-smeared link-variables as illustrated in Fig. 2 a). See also Ref. [2, 3].

The connection between the matrix elements $\tilde{\Phi}^{[\Gamma]}$ and TMDs is established through a parametriza- 
tion in terms of Lorentz-invariant amplitudes $\widetilde{A}_{i}\left(l^{2}, l \cdot P\right)$. For straight Wilson lines, we obtain in analogy to the parametrization in terms of amplitudes $A_{i}\left(k^{2}, k \cdot P\right)$ in Ref. [8] (here our sign conventions follow Ref. [10] with the substitution rule $\left.k \rightarrow i m_{N}^{2} l\right)$ :

$$
\begin{aligned}
\tilde{\Phi}^{\left[\gamma^{\mu}\right]} & =2 P^{\mu} \widetilde{A}_{2}+2 i m_{N}^{2} l^{\mu} \widetilde{A}_{3}, \\
\tilde{\Phi}^{\left[\gamma^{\mu} \gamma^{5}\right]} & =-2 m_{N} S^{\mu} \widetilde{A}_{6}-2 i m_{N} P^{\mu}(l \cdot S) \widetilde{A}_{7}+2 m_{N}^{3} l^{\mu}(l \cdot S) \widetilde{A}_{8}, \\
\tilde{\Phi}^{\left[i \sigma^{\mu v} \gamma^{5}\right]} & =2 P^{[\mu} S^{v]} \widetilde{A}_{9}+2 i m_{N}^{2} l^{[\mu} S^{v]} \widetilde{A}_{10}+2 m_{N}^{2} l^{[\mu} P^{v]}(l \cdot S) \widetilde{A}_{11} .
\end{aligned}
$$

The TMDs are then obtained by

$$
\begin{aligned}
& f_{1}\left(x, \mathbf{k}_{\perp}^{2}\right)=2 \oint \text { fl } \widetilde{A}_{2}\left(l^{2}, l \cdot P\right), \quad h_{1}\left(x, \mathbf{k}_{\perp}^{2}\right)=-2 \oint g\left(t \widetilde{A}_{9 m}\left(l^{2}, l \cdot P\right),\right. \\
& h_{1 L}^{\perp}\left(x, \mathbf{k}_{\perp}^{2}\right)=4 m_{N}^{2} \partial_{\mathbf{k}_{\perp}^{2}}\left(\oint \not c \widetilde{A}_{10}\left(l^{2}, l \cdot P\right)+\partial_{x} \oint \not c \widetilde{A}_{11}\left(l^{2}, l \cdot P\right)\right) \text {, } \\
& g_{1 T}\left(x, \mathbf{k}_{\perp}^{2}\right)=4 m_{N}^{2} \partial_{\mathbf{k}_{\perp}^{2}} \oint f\left(c c \widetilde{A}_{7}\left(l^{2}, l \cdot P\right),\right.
\end{aligned}
$$

with $\widetilde{A}_{9 m} \equiv \widetilde{A}_{9}-\frac{1}{2} m_{N}^{2} l^{2} \widetilde{A}_{11}$. In the equations above, $\mathscr{F}$ only acts on $l \cdot P$, while $\mathscr{H} l$ only acts on $l^{2}$. Thus $x \leftrightarrow l \cdot P$ and $\mathbf{k}_{\perp}^{2} \leftrightarrow l^{2}$ are pairs of conjugate variables. Our Euclidean lattice approach is restricted to the determination of amplitudes $\widetilde{A}_{i}$ for $l^{0}=-i l_{4}=0$, i.e., to the region $l^{2}<0,|l \cdot P| \leq$ $\sqrt{-l^{2}}|\mathbf{P}|$, where $\mathbf{P}$ is the selected three-momentum of the nucleon on the lattice. The limited range in $|l \cdot P|$ prohibits us from a direct evaluation of $\mathscr{F}$. However, first studies of $x$ - and $\mathbf{k}_{\perp}$ - correlations are possible [16,3]. Moreover, $x$-integrated TMDs and densities are directly accessible: Integrating Eq. (1.1) with respect to $x$ removes $\mathscr{F}$ and sets $l \cdot P$ to zero. Correspondingly, the $x$-integral of, e.g., $f_{1}$ becomes $\int_{-1}^{1} d x f_{1}\left(x, \mathbf{k}_{\perp}^{2}\right) \equiv f_{1}^{[1]}\left(\mathbf{k}_{\perp}^{2}\right)=2 \not l \widetilde{A}_{2}\left(l^{2}, 0\right)$. In Fig. 2 b), open symbols correspond to unrenormalized lattice data for $\widetilde{A}_{2}\left(l^{2}, 0\right)$.

To obtain results independent of our lattice spacing $a$ and our lattice action, we must renormalize our data. The Wilson line $\mathscr{U}\left[\mathscr{C}_{l}\right]$ introduces a length dependent renormalization factor $\exp \left(-\delta m \sqrt{-l^{2}}\right)[17,18,19]$. To fix $\delta m$, we follow the strategy of Refs. [20, 21], and match the renormalized static quark potential $V^{\text {ren }}(r)=V(r)+2 \delta m$ to the string potential $V_{\text {string }}=\sigma r-$ $\pi /(12 r)$ [22] at a matching point $r=1.5 r_{0} \approx 0.7 \mathrm{fm}$. In Fig. 3 a), we test the method for several lattice spacings $a$ on four MILC lattices with similar pion masses $m_{\pi} \approx 500 \mathrm{MeV}$. The renormalized lattice data agree very well with each other and are approximated well by the string potential (red dashed curve) near the matching point, indicated by a vertical dashed line. The procedure implements a gauge-invariant renormalization condition that we can formulate as the demand that the static quark potential asymptotically approach a straight line $\sigma r$ through the origin (shown as a red dashed line). In connection with TMDs, we lack at present an interpretation of this renormalization condition as a physical renormalization or factorization scale. In Figure 3 b), we check the applicability of the approach to Wilson lines by plotting $Y_{\text {line }}^{\text {ren }}(l)=-\ln \left(U_{l+a / 2} / U_{l-a / 2}\right) / a+\delta m$, where $U_{l}$ is the expectation value of the color trace of a straight Wilson line of length $l$ evaluated on a Landau gauge fixed ensemble, and where the length dependent renormalization has been carried out with the values $\delta m$ obtained from the static quark potential. Only at short lengths, $l \lesssim 0.25 \mathrm{fm}$, we find significant differences between lattice data from different lattice spacings, a sign of lattice cutoff effects. For our TMD calculations discussed below we exclude data obtained in this region 
a)

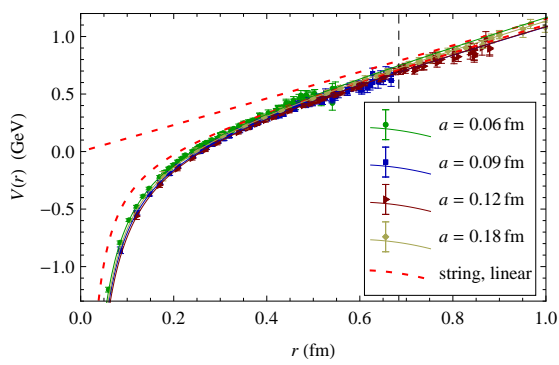

b)

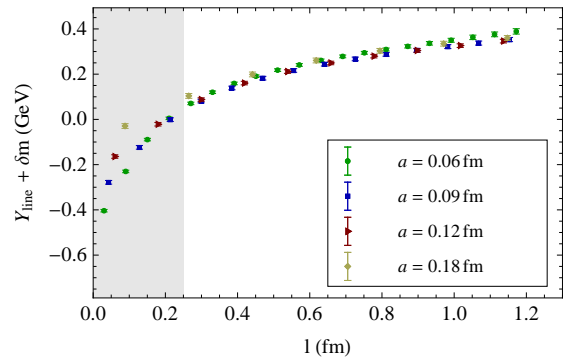

Figure 3: a) Static quark potential from MILC lattices at several lattice spacings $a$, matched to the string potential at $r \approx 0.7 \mathrm{fm}$. b) Test of the renormalization procedure with straight Wilson lines on a gauge fixed ensemble.

from our fits. For $l \gtrsim 0.25 \mathrm{fm}$, we assume that renormalization of the lattice operator can be carried out as in the continuum, $O^{\text {ren }}=Z_{\Psi, z}^{-1} \exp \left(-\delta m \sqrt{-l^{2}}\right) O$, where the renormalization constants $Z_{\Psi, z}^{-1}$ and $\delta m$ are independent of the Dirac structure $\Gamma$ [18].

Figure $2 \mathrm{~b}$ ) shows the renormalized lattice data for $\widetilde{A}_{2}\left(l^{2}, 0\right)$ as solid data points. The curve and statistical error band correspond to a Gaussian fit to this data in the range $\sqrt{-l^{2}} \geq 0.25 \mathrm{fm}$. Note that the renormalization constant $Z_{\Psi, z}^{-1}$ has been fixed (in the isovector, $u-d$-channel) such that the $x$ - $\mathbf{k}_{\perp}$-integrated Gaussian density of unpolarized quarks yields the correct total number of valence quarks, $\int d^{2} \mathbf{k}_{\perp} f_{1, u-d}^{[1]}=1$. Similar fits for the other amplitudes $\widetilde{A}_{10}$, and $\widetilde{A}_{7}$ enable us to calculate the "worm-gear" functions $g_{1 T}^{[1]}, h_{1 L}^{\perp[1]}$, and correspondingly, the dipole deformed $x$-integrated densities $\rho_{T L}^{(q)[1]}$ and $\rho_{L T}^{(q)[1]}$ defined in Eqns. (1.2), (1.3) and shown in Fig. 1 b). While the widths of our distributions depend strongly on our renormalization condition for $\delta m$, average transverse quark momenta can be expressed in terms of ratios of the Gaussian amplitudes at $l^{2}=0$ :

$$
\begin{aligned}
& \left.\left\langle\mathbf{k}_{x}\right\rangle_{T L} \equiv \frac{\int d^{2} \mathbf{k}_{\perp} \mathbf{k}_{x} \rho_{T L}^{[1]}}{\int d^{2} \mathbf{k}_{\perp} \rho_{T L}^{[1]}}\right|_{\substack{\lambda=1, \mathbf{S}_{\perp}=(1,0)}}=-m_{N} \frac{\widetilde{A}_{7}(0,0)}{\widetilde{A}_{2}(0,0)}=\left\{\begin{array}{c}
67(5) \mathrm{MeV} \text { (up) } \\
-30(5) \mathrm{MeV} \text { (down) }
\end{array}\right. \\
& \left.\left\langle\mathbf{k}_{x}\right\rangle_{L T} \equiv \frac{\int d^{2} \mathbf{k}_{\perp} \mathbf{k}_{x} \rho_{L T}^{[1]}}{\int d^{2} \mathbf{k}_{\perp} \rho_{L T}^{[1]}}\right|_{\substack{\Lambda=1, \mathbf{s}_{\perp}=(1,0)}}=-m_{N} \frac{\widetilde{A}_{10}(0,0)}{\widetilde{A}_{2}(0,0)}=\left\{\begin{array}{r}
-60(5) \mathrm{MeV} \text { (up) } \\
16(5) \mathrm{MeV} \text { (down) }
\end{array}\right.
\end{aligned}
$$

(errors statistical only). In these ratios, renormalization factors largely cancel. Reference [23] reveals a remarkable similarity of our results with a light-cone constituent quark model [24], despite the unphysically large quark masses employed in our lattice calculation: They find $\left\langle\mathbf{k}_{x}\right\rangle_{T L}=$ $-\left\langle\mathbf{k}_{x}\right\rangle_{L T}=55.8 \mathrm{MeV}$ for up-, and $\left\langle\mathbf{k}_{x}\right\rangle_{T L}=-\left\langle\mathbf{k}_{x}\right\rangle_{L T}=-27.9 \mathrm{MeV}$ for down-quarks.

\section{Staple shaped gauge links}

TMDs for SIDIS or the Drell-Yan process, in particular T-odd distributions like the Sivers function $f_{1 T}^{\perp}$ in Eq. (1.2), require gauge links out to infinity in a direction $v$, see Fig. 4 a). To avoid rapidity divergences, the direction $v$ can be chosen slightly off the light cone, see, e.g., [25, 26]. The amplitudes $\widetilde{A}_{i}$ and the TMDs then also depend on Lorentz-invariant structures involving $v$, in particular on the parameter $\zeta \equiv(2 v \cdot P)^{2} / v^{2}$, with respect to which the TMDs follow an evolution 
a)

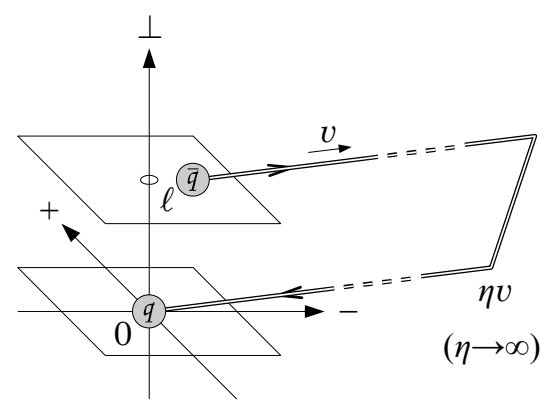

b)

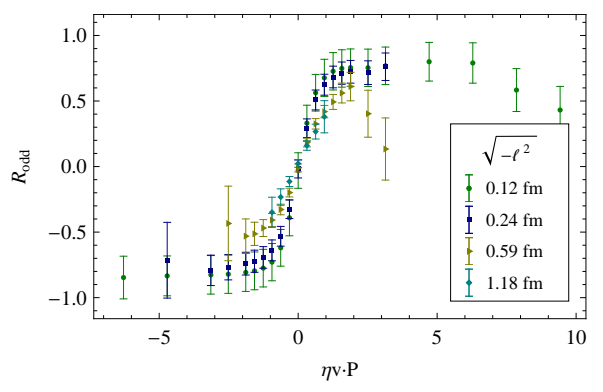

Figure 4: a) Staple shaped Wilson line. b) Test calculation of a T-odd ratio of amplitudes using staple shaped links at $m_{\pi} \approx 800 \mathrm{MeV}$.

equation [25]. Our lattice operators are restricted to spacelike staple directions, $v^{0}=0$, i.e., our amplitudes are limited to $0 \leq-\zeta \leq|2 \mathbf{P}|^{2}$. We can only realize staples of finite extent $\eta v$, but we can look for a plateau at large $\eta$. Remaining challenges regarding the definition of TMDs mainly relate to the regularization of divergences attributed to the Wilson line and possible soft-factor modifications of Eq. (1.1) needed to ensure QCD factorization. First lattice studies are planned for ratios of amplitudes, in which renormalization factors and potential soft factors cancel. Figure 4 b) shows results from a test calculation [2] of a ratio of T-odd over T-even amplitudes $R_{\text {odd }} \equiv$ $\left(\widetilde{A}_{12}-\left(m_{N} /|\mathbf{P}|\right)^{2} \widetilde{B}_{8}\right) / \widetilde{A}_{2}$, evaluated at $l \cdot P=0,|\mathbf{P}| \approx 0.5 \mathrm{GeV}$, for selected values of $l^{2}$ and a range of staple extents $\eta$. Note that $\widetilde{A}_{12}$ would correspond to the Sivers function $f_{1 T}^{\perp}$ for lightlike $v$, while the amplitudes $\widetilde{B}_{i}$ parametrize explicit $v$-dependence [10]. As expected, the result is an odd function of $\eta v \cdot P$. Moreover, we see the onset of a plateau at $|\eta v \cdot P| \gtrsim 2$. This is a promising indication that lattice estimates could be feasible for, e.g., the transverse momentum shift $\left\langle\mathbf{k}_{y}\right\rangle_{T U}$ due to the Sivers function.

\section{Conclusions}

We have performed first lattice studies of TMDs using non-local operators with a simplified, straight gauge link. Resulting average momentum shifts $\left\langle\mathbf{k}_{x}\right\rangle_{T L}$ and $\left\langle\mathbf{k}_{x}\right\rangle_{L T}$ corroborate model results. Staple-shaped gauge links can potentially address TMDs specific to SIDIS or the DrellYan process, including T-odd functions responsible for single-spin asymmetries.

\section{Acknowledgments}

We are very grateful to the LHP and MILC collaborations, for providing us gauge configurations and propagators. We thank Vladimir Braun, Meinulf Göckeler, Gunnar Bali, Markus Diehl, Alexei Bazavov, and Dru Renner for very helpful discussions. For our calculations, we have been using computing resources at Jefferson Lab. Our software uses the Chroma-library [27], and we use USQCD computing resources at Jefferson Lab. We acknowledge support by the Emmy-Noether program and the cluster of excellence "Origin and Structure of the Universe" of the 
DFG (Ph.H. and B.M.), SFB/TRR-55 (A.S.) and the US Department of Energy grant DE-FG0294ER40818 (J.N.). Authored by Jefferson Science Associates, LLC under U.S. DOE Contract No. DE-AC05-06OR23177. The U.S. Government retains a non-exclusive, paid-up, irrevocable, world-wide license to publish or reproduce this manuscript for U.S. Government purposes.

\section{References}

[1] P. Hägler, B. U. Musch, J. W. Negele, and A. Schäfer, Europhys. Lett. 88 (2009) 61001.

[2] B. U. Musch. PhD thesis, TU München, 2009. arXiv:0907.2381. http://nbn-resolving.de/urn/resolver.pl?urn:nbn:de:bvb:91-diss-20090529-738021-1-8.

[3] B. Musch, P. Hägler, A. Schäfer, and J. Negele. tbp.

[4] P. Hägler, Phys. Rept. 490 (2010) 49-175.

[5] J. Collins, PoS LC2008 (2008) 028.

[6] I. O. Cherednikov, A. I. Karanikas, and N. G. Stefanis, Nucl. Phys. B840 (2010) 379-404.

[7] J. C. Collins and D. E. Soper, Nucl. Phys. B194 (1982) 445.

[8] P. J. Mulders and R. D. Tangerman, Nucl. Phys. B461 (1996) 197-237.

[9] D. Boer and P. J. Mulders, Phys. Rev. D57 (1998) 5780-5786.

[10] K. Goeke, A. Metz, and M. Schlegel, Phys. Lett. B618 (2005) 90-96.

[11] J. C. Collins, Phys. Lett. B536 (2002) 43-48.

[12] P. Hägler et al., LHPC collaboration, Phys. Rev. D77 (2008) 094502.

[13] C. W. Bernard et al., Phys. Rev. D64 (2001) 054506.

[14] C. Aubin et al., Phys. Rev. D70 (2004) 094505.

[15] A. Bazavov et al., Rev. Mod. Phys. 82 (2010) 1349-1417.

[16] B. U. Musch et al., PoS LC2008 (2008) 053.

[17] N. S. Craigie and H. Dorn, Nucl. Phys. B185 (1981) 204.

[18] H. Dorn, Fortsch. Phys. 34 (1986) 11-56.

[19] P. Boucaud, C. L. Lin, and O. Pene, Phys. Rev. D40 (1989) 1529.

[20] M. Cheng et al., Phys. Rev. D77 (2008) 014511.

[21] A. Bazavov et al., Phys. Rev. D80 (2009) 014504.

[22] M. Lüscher, K. Symanzik, and P. Weisz, Nucl. Phys. B173 (1980) 365.

[23] B. Pasquini, S. Boffi, A. V. Efremov, and P. Schweitzer, arXiv:0912.1761 [hep-ph].

[24] B. Pasquini, S. Cazzaniga, and S. Boffi, Phys. Rev. D78 (2008) 034025.

[25] J. C. Collins and D. E. Soper, Nucl. Phys. B193 (1981) 381.

[26] X.-d. Ji, J.-p. Ma, and F. Yuan, Phys. Rev. D71 (2005) 034005.

[27] R. G. Edwards and B. Joo, SciDAC collaboration, Nucl. Phys. Proc. Suppl. 140 (2005) 832. 